\title{
Sensor Spatial Distortion, Visual Latency, and Update Rate Effects on 3D Tracking in Virtual Environments
}

\author{
S.R. Ellis, B.D. Adelstein ${ }^{1}$, S. Baumeler' ${ }^{2}$ G.J. Jense ${ }^{3}$, R.H. Jacoby ${ }^{4}$ \\ NASA Ames Research Center
}

${ }^{1}$ UC Berkeley, ${ }^{2}$ ETH Zurich, ${ }^{3} \mathrm{TNO},{ }^{4}$ Sterling Software

\begin{abstract}
We examined the effects on human $3 D$ tracking performance of several common defects of immersing virtual environments: spatial sensor distortion, visual latency and low update rates. Results show: 1) Removal of relatively small static distortion had minor effects on tracking accuracy, 2) An Adapted Cooper-Harper controllability scale proved the most sensitive subjective indicator of simulation degradation, and 3) RMS tracking error and subjective impressions were more influenced by changing visual latency than by update rate.
\end{abstract}

\section{Introduction}

Virtual environments are personal simulators. Their usefulness as tools for training, perceptual motor research, interpersonal communication, data visualization, or many other purposes depends upon the accurate presentation of environmental stimuli and recording of user interaction. Virtual environments that have been used for military aircraft simulation have been engineered to high standards of static and dynamic fidelity (CAE and Kaiser SIM-eyes). But these systems are very expensive. Users of the more moderately priced systems must still contend with significantly inadequate static and dynamic system characteristics. (e.g., [1], [2], [5], [6], [7])

The following experiment examines the impact on human performance of several common system defects that we have removed or are in the process of minimizing in our virtual environment simulations. We have examined sensor spatial distortion, visual latency and update rate variation. Performance during large amplitude 3D tracking is assessed in the presence and absence of these defects by objective and subjective methods. These methods allow performance trade-offs to be measured and point toward improved future assessment techniques.

\section{Experiment}

\subsection{Methods}

This study used the same large amplitude 3D tracking technique employed previously under conditions in which the precision of interactivity as the environmental simulation was degraded by reduction of update rate and increases in latency [1]. In this task, subjects immersed in a simple virtual environment simulation of a room are asked to keep a tetrahedron within a cube which is driven by a modified sum of sines forcing function to move irregularly within the virtual space. The cube moved within a volume that was between shoulder level and arms reach above and in front of each subject, but with sufficient lateral displacement to require subjects to take several steps forwards and backwards to perform the task. The tracking difficulty was adjusted to be relatively easy so that measurement of degradation of simulation fidelity would not be compromised by performance saturation effects, i.e., normalized RMS tracking error was $\sim 0.15$ for easiest conditions. Fig. 1 illustrates the tracking task.
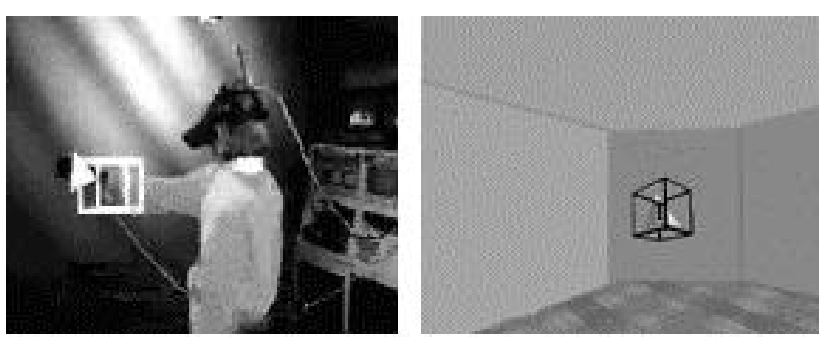

Fig. 1. The left panel shows a subject performing the 3D tracking task by attempting to keep the tetrahedron inside the moving cube. The subject's actual view through the head-mounted display is represented by a screen image in the middle panel.

The environmental simulation was produced using World Tool Kit on a SGI Skywriter graphics computer with RE-1 graphics. The room was displayed in full color stereo to mobile users wearing a VR4 head-mounted display. FasTrak hand and head position sensors were used with a custom parallel interface driver [3].

It should be noted that our display rates were clock-stabilized, accounting for the fact that our fastest stereo display rate was limited to $20 \mathrm{~Hz}$. In one experimental condition the orientation and position distortions present in our FasTrak were corrected using an adaptation of an algorithm developed by Kenwright and Lane [4]. Fig. 2 shows some of our measurements of the position distortion error in our calibrated volume. Position correction was essentially 
complete at each measurement node, as shown by the rectified data of the outermost shell of measurements (darker lines) within the distorted volume. Residual position errors at intermediate (non-nodal) locations were reduced approximately sevenfold by the correction procedure. For most of our data, error in orientation was measured by the quaterion that would rotate the measured local vertical, approximately a surface normal to each quadrilateral patch (e.g. shaded region in Fig. 2), into true vertical. These orientation errors within each calibrated cell were corrected by inverse rotations based on error measurements at adjacent calibration grid nodes.

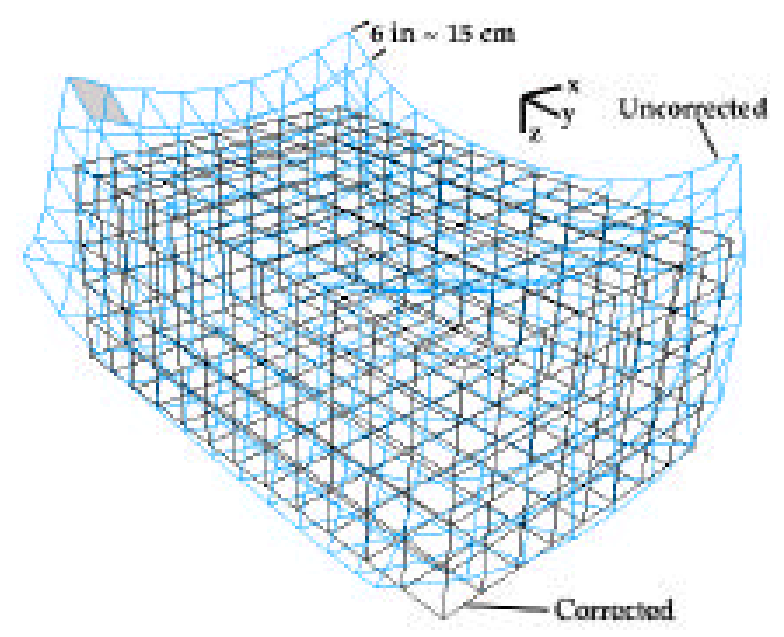

Fig. 2. The lighter 3D mesh shows the position distortion measured within our calibrated volume. For clarity of presentation, only part of the calibrated volume is shown.

\subsection{Tracking task}

As in previous experiments [1], the tracking task was presented for two minute intervals, with the first 10 seconds of the tracking data ignored as a warm-up period. Due to discrete frame rates and our desire for clock stabilized rates, it was not possible to experimentally cross frame rates with latency. For the three frame rate $(6,12,20 \mathrm{~Hz})$ and five latency categories ${ }^{1}(480,320,230,130,80 \mathrm{msec})$ used, we were able to isolate 11 conditions (see Fig. 3).

Each of these conditions was presented three times consecutively followed by a pause during which questionnaire data were collected. The questionnaire included an adapted Cooper-Harper controllability scale, and seven bipolar adjectival scales measuring subjective realism, environmental stability, subject's dizziness, nausea, neck ache, head/eye ache, and eye tearing [1].

\footnotetext{
${ }^{1}$ Categories were used because specific latency could not be reproduced exactly for each update rate. Accuracy was $\pm 20 \%$ about the central value for each category. Latencies were calculated from a model in [3].
}

\subsection{Experimental Design}

Each group of three runs was repeated with random selections of latency and frame rate until all 11 of these conditions were used. This grouping produced a block which was repeated with separate randomization of the latency/frame rate condition six times for each subject: three times with spatial distortion correction and three times without distortion correction. Each group of three blocks with and without distortion was usually presented on separate days. Subjects were given the opportunity to take rest periods, sometimes extending to the next day, between blocks of the experiment. Five subjects began with distortion correction and five began without the correction. All subjects were male and were either laboratory personnel or provided from the NASA Ames/Bionetics paid subject pool.

Repeated-measures ANOVA were calculated for the separate dependent measures, normalized RMS ( $n R M S)$ tracking error, Adapted Cooper-Harper $(A C H)$ controllability scale, and the bipolar adjectival scales of realism, stability, dizziness, nausea, neck ache, head/eye ache, eye tearing: Dynamic Display Conditions (11) X Distortion (2) X Order (2) with 5 subjects nested in each Order group.

\section{Results}

For brevity, only ANOVAs on the variables that were statistically significant in the previous experiment [1] will be considered. Because of multiple independent ANOVAs, only statistically robust $(p<.05)$ are reported.

The presence or absence of the distortion correction had no effect on $n R M S(\mathrm{~F}(1,8)=0.51$, n.s. $)$, subjective Realism $(F(1,8)=1.10$, n.s. $)$, or subjective stability $(F(1,8)=$ 0.42 , n.s.). The distortion correction showed a significant main effect on the $A C H$ Scale $(F(1,8)=8.945, p<.017)$, reducing the rating from 3.27 to 2.98 . No other statistical tests showed effects purely attributable to correction of the spatial distortion.

Fig. 3 shows performance measures in this experiment for the variables that proved statistically significant in the prior study [1]. The results generally replicate the previous experiment, but here subjectively judged realism was not reliably affected by the degradation of latency and frame rate. Most strikingly, the previously observed similarity of the $A C H$ scale reports and the $n R M S$ measurement was replicated. Though subjectively judged stability of the virtual objects and world was significantly affected by the changes of frame rate and latency, its pattern of results does not match the shape of the $n R M S$ results as closely as the Cooper-Harper results.

Rather than estimate data for the cells which we could not test in order to compute an interaction between latency and frame rate, the possibility of an interaction was investigated by multiple regression on the raw observations, predicting $n R M S, A C H$ and Stability from latency, frame rate, and latency crossed with frame rate. None of the three 


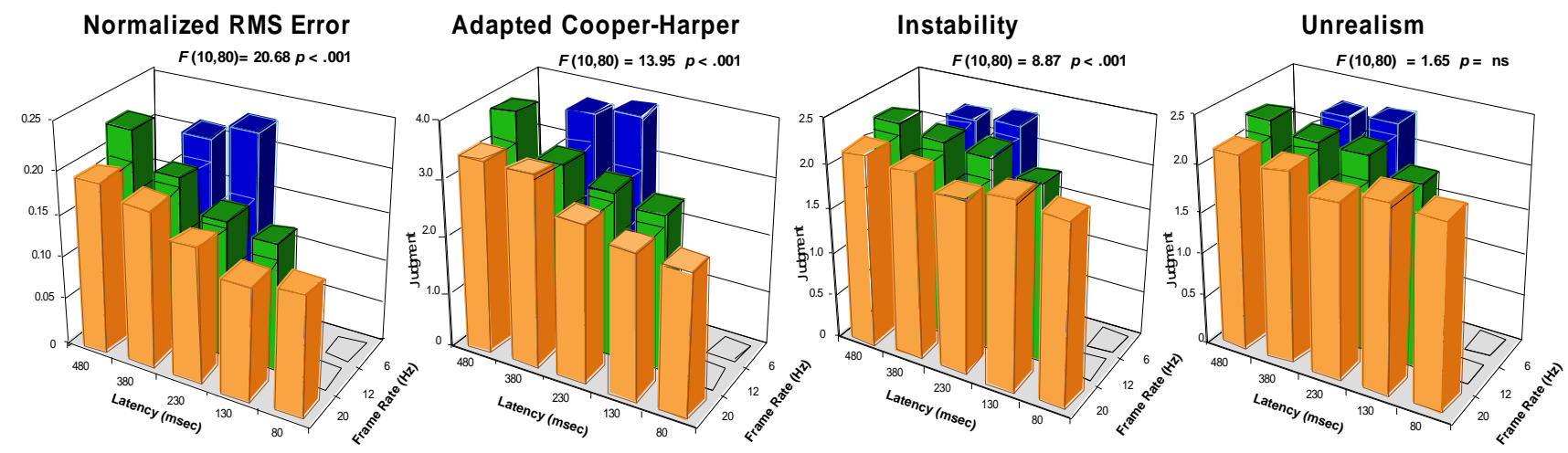

Fig. 3. Principal results from the analyses of variance. Realism and stability were inverted so that positive values on vertical axes correspond to poorer performance of fidelity judgments.

equations (Table 1) have a statistically significant interaction term. Latency rather than frame rate generally shows greater strength and statistical reliability.

\begin{tabular}{|c|c|c|c|c|}
\hline \multicolumn{5}{|c|}{$N=660 ; R=0.55 ; F(3,656)=94.49 ; \quad p<.0001$} \\
\hline Variable & Std Error & Std Coef & t-value & Prob \\
\hline lat & $1.30 \mathrm{E}-5$ & 0.49 & 14.72 & 0001 \\
\hline$f \_r$ & $2.24 \mathrm{E}-3$ & -0.35 & 1.55 & 1215 \\
\hline lat $* f_{-} r$ & $8.05 \mathrm{E}-5$ & 0.16 & 0.71 & 4772 \\
\hline \multicolumn{5}{|c|}{$\begin{aligned} \boldsymbol{A C H}=3.01+ & .00279 * \text { lat }-.08 * f_{-} r \\
& +.00171 * \text { lat } * f_{-} r \\
660 ; R=0.55 ; & F(3.656)=93.46 ; \quad p<.00\end{aligned}$} \\
\hline Variable & Std Error & Std Coef & t-value & Prob \\
\hline lat & $1.91 \mathrm{E}-4$ & 0.48 & 14.58 & .0001 \\
\hline$f \_r$ & 0.03 & -0.52 & 2.28 & 0228 \\
\hline lat $* f_{-} r$ & $1.19 \mathrm{E}-3$ & 0.33 & 1.44 & 1504 \\
\hline$N=660 ; R=0$ & \multicolumn{4}{|c|}{$\begin{aligned}= & 2.76+.00274 * \text { lat }-.14 * f_{-} r \\
& +.00325 * \text { lat } * f_{\_} r\end{aligned}$} \\
\hline Variable & Std Error & \begin{tabular}{|l} 
Std Coef \\
\end{tabular} & t-value & Prob \\
\hline lat & $2.68 \mathrm{E}-4$ & 0.36 & 10.21 & 0001 \\
\hline$f_{-} r$ & 0.05 & -0.71 & 2.93 & 0036 \\
\hline lat $* f_{-} r$ & $1.66 \mathrm{E}-3$ & $1.66 \mathrm{E}-3$ & 1.95 & 0512 \\
\hline
\end{tabular}

Table 1. Multiple regression for $n R M S, A C H$, and Stability testing relative strengths of independent variables latency (lat) and frame rate $\left(f \_r\right)$ and their interaction.

A correlation matrix (Table 2) of all dependent and independent measures was calculated to match that reported in the previous experiment. This table shows that the two independent variables of frame rate and latency correlated significantly only with the $A C H$ scale, the subjective stability judgment, and the $n R M S$ tracking error. In all cases the strengths of the correlations with latency were stronger than those with frame rate, supporting the multiple regres- sions described earlier. A number of the correlations among the dependent variables were also significant, suggesting the eventual possibility of a factor analysis.

\section{Discussion}

\subsection{Impact of distortion}

The absence of a major impact of the correction to the spatial sensor distortion on human performance might seem surprising, but can be understood in terms of the error feedback in the tracking task. From the viewpoint of the operators, the distortion manifests itself as additional displacements of the tetrahedral cursor controlled by their hand position. These displacements cause uncommanded tracking errors to occur with respect to the moving target cube. However, because the operators cannot see their real hands in the virtual environment, they cannot easily distinguish the cause of the uncommanded errors. These may be attributed to movement of their cursor with respect to their hand. In any case, the additional error is not large with respect to their hand movements. It is introduced smoothly. They can see it and they can act to correct it.

The distortion also acts on the head measurement. Any error in this action displaces and rotates the entire virtual environment; it does not affect the relative position of the cursor or the target.

The distortion, however, was apparently not totally negligible since its presence increased $A C H$ scale. This effect is not surprising since the Cooper-Harper scale is often sensitive to workload effects and could be providing evidence for increased tracking effort. But even for this measure, the effect of removal of the distortion is small.

It is important to note, however, that the small effects seen in this experiment would very likely grow significantly for see-through display tasks that depend upon correct spatial registration with the external world. Additionally, as is evident from Fig. 2, the magnitude of the distortion in our tracked volume is relatively small, since 


\begin{tabular}{|c|c|c|c|c|c|c|c|c|c|c|c|}
\hline \multirow[b]{2}{*}{ Frame } & Frame & Latency & $\mathrm{ACH}$ & Realism & Stability & Dizziness & Nausea & Neckache & Head/eye ache & Eye tearing & nRMS \\
\hline & 1.000 & & & & & & & & & & \\
\hline \multirow{2}{*}{$\begin{array}{l}\text { Latency } \\
\mathrm{ACH}\end{array}$} & -.153 & 1.000 & & & & & & & & & \\
\hline & -.320 & .607 & 1.000 & & & & & & & & \\
\hline \multirow{2}{*}{$\begin{array}{l}\text { Realism } \\
\text { Stability }\end{array}$} & -.057 & .060 & .202 & 1.000 & & & & & & & \\
\hline & -.339 & .451 & .688 & .520 & 1.000 & & & & & & \\
\hline Dizziness & -.035 & .007 & .247 & -.014 & .155 & 1.000 & & & & & \\
\hline \multirow{5}{*}{$\begin{array}{l}\text { Nausea } \\
\text { Neckache } \\
\text { Head/eye ache } \\
\text { Eye tearing } \\
\text { nRMS }\end{array}$} & -.028 & -.043 & -.020 & -.017 & .001 & .656 & 1.000 & & & & \\
\hline & -.071 & -.032 & .155 & -.147 & .158 & .653 & .556 & 1.000 & & & \\
\hline & -.049 & .033 & .349 & .107 & .376 & .381 & .371 & .682 & 1.000 & & \\
\hline & -.121 & .007 & .100 & -.015 & .157 & .638 & .704 & .754 & .630 & 1.000 & \\
\hline & -.349 & .678 & .539 & .167 & .329 & -.144 & -.108 & -.298 & -.073 & -.097 & 1.000 \\
\hline
\end{tabular}

110 observations used in this computation.

Table 2. Correlation matrix of dependent and independent variables with statistical test keeping overall $\alpha<.05$. We compute $\alpha$ for 55 independent tests. The $d f$ for each correlation is based on 11 observations/subject, yielding nine $d f$ per subject Eleven subjects give a total $d f=99$, (Critical $r=.341$ for $p<.001$. Significant correlations in boldface).

we have been careful to remove objects that produce large disturbances. Distortion from such objects could easily change our results.

\subsection{Replication of latency and update effects}

The subjective and objective measurements of tracking performance substantially replicate the findings in the previous experiment [1] which framed the current investigation. The $A C H$ scale proved better correlated with the objective tracking performance than the bipolar adjectival scales which were generally not as sensitive to the reduction in simulation fidelity caused by dropping frame rates or increased visual latency.

As in the previous experiment, latency, in particular, appeared to be the stronger, more statistically reliable factor degrading performance. It is important to note, however, that we were able to experimentally vary latency over a proportionately greater range than update rate (80-480 msec, i.e., 1:6 vs. 6-20, i.e., 1:3.7), This difference in itself cannot account for the differences in correlations since the smaller number of levels of frame rate (3) versus the number of levels of latency (5) would have the opposite effect, tending to increase the correlations associated with frame rate effects. The statistical analyses ideally control for these differences but they cannot substitute for an explicit factorial test examining latency and frame rate over more similar ranges. We have recently determined that we can now conduct such an experiment with new equipment that could potentially stabilize updates at $60 \mathrm{~Hz}$. Future investigations should, accordingly, provide additional information on the relative importance of frame rate and latency for performance in virtual environments. It is probably worth noting that the manufacturers of high performance computer graphics systems currently sacrifice latency for frame rate. The present findings suggest they could improve their systems' interactivity were they to alter their existing trade-off.

Acknowledgments We thank N.S. Dorighi, B.M. Menges, M.J. Young, D. Delgado, and D.N. Kenwright for their assistance.

\section{References}

[1] S.R. Ellis, N.S. Dorighi, B.M. Menges, B.D. Adelstein, and R H. Jacoby, "In search of equivalence classes in subjective scales of reality," in M.J. Smith, G. Salvendy, and R.J. Koubec, eds., Design of Computing Systems, Advances in Human Factors Ergonomics, vol $21 B$, pp. 873-876. Elsevier, 1997

[2] W. Barfield and C. Hendrix, "The effect of update rate on the sense of presence within virtual environments," Virtual Reality: Res., Dev., Appl., 1(1), 3-15, 1995.

[3] R.H. Jacoby, B.D. Adelstein, and S.R. Ellis, "Improved temporal response in virtual environments through system hardware and software reorganization," Proc., SPIE 2653, pp. 271-284, 1996.

[4] D.N. Kenwright and D.A. Lane, "Interactive timedependent particle tracing using tetrahedral decomposition," IEEE Trans. Visualiz. Comp. Graph., 2(2), 120129, 1996.

[5] A. Liu, G. Tharp, L. French, S. Lai, and L. Stark, "Some of what one needs to know about using head mounted displays to improve teleoperator performance," IEEE Trans. Rob. Autom., 9(5), 638-648, 1993.

[6] C. Ware and R. Balakrishnan, "Reaching for Objects in VR Displays: Lag and Frame Rate," ACM Trans. Comp. Hum. Interact., 1(4) 331-356, 1994.

[7] B. Watson, V. Spaulding, N. Walker, and W. Ribarsky, "Evaluation of the Effects of Frame Time Variation on VR Task Performance," Proc., Virtual Reality Ann. Internat. Symp., pp. 38-44, Albuquerque, NM, 1997. 